\title{
TRaDe: Data Race Detection for Java
}

\author{
Mark Christiaens $^{1}$ and Koen De Bosschere ${ }^{1}$ \\ ELIS, Ghent University, \\ Sint-Pietersnieuwstraat 41, 9000 Gent, Belgium, \\ $\{$ mchristi, kdb\}@elis.rug.ac.be
}

\begin{abstract}
This article presents the results of a novel approach to race detection in multi-threaded Java programs. Using a topological approach to reduce the set of objects needing to be observed for data races, we have succeeded in reducing significantly the time needed to do data race detection. Currently, we perform data race detection faster than all known competition. Furthermore, TRaDe can perform data race detection for applications with a high, dynamically varying number of threads through the use of a technique called 'accordion clocks'.
\end{abstract}

\section{Introduction}

The technique of multi-threaded design has become a permanent part of almost every serious programmer's toolbox. When applying a multi-threaded design to a programming task, a task is split into several threads. Each of these subtasks can be executed on a separate processor, increasing parallelism and speed. A group of threads can be dedicated to respond to external events, so a very fast response time can be obtained using this design.

Together with the advantages of multi-threaded design, a new type of bug has appeared which is very hard to handle: a data race. As each thread performs its task in the program, it modifies data structures along the way. It is up to the programmer to ensure that the operations of threads happen in an orderly fashion. If the threads do not behave properly, but modify a data structure in a random order, a data race occurs. An example of a data race can be seen in Figure 1

Data Races are very hard to locate. They are non-deterministic, since their occurrence is dependent on the execution speed of the threads involved. On top of that, data races are non-local. Two pieces of code whose only relation is that they both utilize the same data structures, independently can function perfectly but when executed simultaneously, they will wreak havoc. Finding or even suspecting a data race can take days of debugging time.

\section{State of the Art in Data Race Detection}

In general, finding data races is at least an NP-hard problem [9], so usually, techniques are proposed to detect data races in one particular execution and 


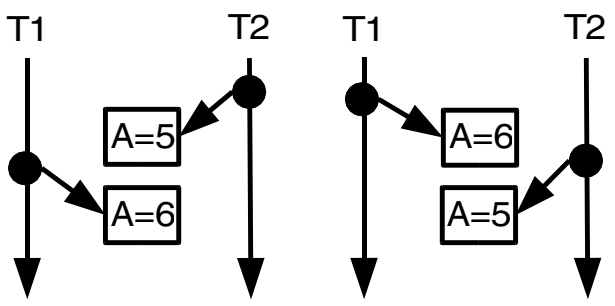

Fig. 1. On the left we see thread 2 accessing a common object, $A$, and writing the value 5. This is followed by thread 1, writing the value 6 to A. The result of this operation is that A contains the value 6 . On the right, thread 1 for some reason executes faster which results in the same events happening but in reverse order. This is possible since there is no synchronisation between thread 1 and 2. A now contains the value 5 .

not in all possible executions of a program. Several systems for detecting data races for general programs have been devised [1011. These techniques can find some types of data races (their definitions of data race differ) but have the disadvantage of being very time consuming. A time overhead of a factor of 30 is not uncommon. This time overhead is caused by the fact that every read and write operation performed by the threads is observed.

Recently, modern programming languages with a more object oriented philosophy have seen the light. One of these is the Java programming language designed by Sun [813] at the beginning of the 1990s. Java was designed with multi-threading in mind. Several language constructs are dedicated to the manipulation of threads. Since it is so easy to make a multi-threaded program in Java, the data race problem manifests itself even more frequently than in the usual C-programs.

It is therefore no coincidence that for this language, already two tools exists that detect data races in particular executions of Java programs: AssureJ and JProbe [6.7]. Here too, the problem of overhead pops up. A time overhead of at least a factor 10 is common. This is smaller than for general programs because each thread in Java has a local stack. The values on this stack can only be manipulated by the thread owning the stack. Therefore all read and write operations performed on this stack need not be observed to find data races.

\section{Topological Race Detection}

Java has a specific property that can be exploited to make race detection even more efficient. The only way an object can be manipulated is through a reference to that object. Pointers do not exist so the problem that through a pointer every location in memory can be modified, does not exist.

It is therefore possible to determine exactly which objects can be reached by a thread at a certain point in an execution and which cannot. Objects that are 
reachable by only one thread cannot be involved in a data race since no other thread can simultaneously be modifying that object.

In Java we have exploited this fact as follows. Initially, at an object's construction through invoking one of the bytecodes new, newarray, anewarray and multianewarray, the only reference to it is stored on the stack of the thread creating the object. No other threads can access this reference, therefore at this point this object cannot be involved in a data race.

From that point forward, this reference can be manipulated by the thread, maybe even be stored in an object or a class. We have devised a system, called TRaDe (Topological RAce Detection), to detect dynamically when an object becomes reachable by more than one thread i.e. the object becomes global. It constantly observes all manipulations of references which alter the interconnection graph of the objects used in a program (hence the name "topological" of the method).

Using this classification mechanism, we can detect data races more efficiently. Only for objects that are reachable by more than one thread, is full data race detection necessary. This means that we save execution time and memory by not analyzing the read and write operation to these objects and by not maintaining data structures for race detection. During the last year we have implemented our ideas by modifying the interpreter (and shutting down the JIT compiler) contained in the source of the JDK1.2.1 from Sun so that it detects data races. To measure the performance of this implementation, we collected a set of applications consisting of a number of large programs to be used as a benchmark (see Table 1).

Table 1. Description of the Java benchmarks used

\begin{tabular}{l|l} 
Name & Description \\
\hline SwingSet & A highly multi-threaded demo of the Swing widget set \\
Jess & A clone of the expert system shell CLIPS \\
Resin & Web server entirely written in Java \\
Colt & Open source libraries for high performance scientific and technical computing \\
Raja & Ray tracer
\end{tabular}

In Table 2 we have measured the execution time and memory consumption of the benchmarks while doing race detection with TRaDe, AssureJ and JProbe. For comparison, we have added the execution time when executing with a modern JIT compiler, the Hotspot 1.0.1 build $f$ and with the interpreter version on which TRaDe itself is based.

The benchmarks were performed on a Sun Ultra 5 workstation with 512 MB of memory and a $333 \mathrm{MHz}$ UltraSPARC IIi with a $16 \mathrm{~KB}$ L1 cache and a $2 \mathrm{MB}$ L2 cache. JProbe could not complete all benchmarks. In this case, the benchmarks were run on a larger system to get an indication of JProbe's performance. The 
larger system was a Sun Enterprise 450 with 2048 MB of memory, 4X400 Mhz UltraSPARC IIi processors with $16 \mathrm{~KB}$ L1 direct mapped cache and 4 MB L2 direct mapped cache. These measurements are indicated with a $\dagger$.

As can be seen in Table 2, using TRaDe, we can outperform all known competition. TRaDe is approximately a factor 1.6 faster than AssureJ, the fastest competition. AssureJ is the closest competitor in terms of speed and usually beats us in terms of memory consumption. We must note however that we have found AssureJ not to detect all data races. When a thread is being destroyed, AssureJ apparently prematurely removes all information relating to this thread although future activities of other threads can still cause a data race with activities of this thread.

Table 2. Execution time and memory consumption of the Java benchmarks. At the bottom, averages and normalized averages (with $\mathrm{TRaDe}=1$ ) are given for all systems except JProbe who couldn't perform all benchmarks.

\begin{tabular}{l|cc|cc|cc|cc|cc|} 
& \multicolumn{2}{|c|}{ TRaDe } & \multicolumn{2}{|c|}{ AssureJ } & \multicolumn{2}{|c|}{ JProbe } & \multicolumn{2}{|c|}{ HotSpot } & \multicolumn{2}{|c|}{ Interpreter } \\
& $\mathrm{s}$ & $\mathrm{MB}$ & $\mathrm{s}$ & $\mathrm{MB}$ & $\mathrm{s}$ & $\mathrm{MB}$ & $\mathrm{s}$ & $\mathrm{MB}$ & $\mathrm{s}$ & $\mathrm{MB}$ \\
\hline SwingSet & 98.3 & 126.6 & 160.6 & 73.3 & $>1200 \dagger$ & $>650 \dagger$ & 20 & 41.8 & 15 & 29.8 \\
Jess & 370.3 & 12.1 & 610 & 17.5 & $>3600 \dagger$ & $>650 \dagger$ & 22 & 19.8 & 76 & 8 \\
Resin & 56.5 & 27.6 & 68 & 27.3 & 193 & 226.17 & 11.8 & 27.9 & 10 & 13.7 \\
Colt & 132.5 & 25 & 187.8 & 21.2 & 471.6 & 71 & 27.8 & 23.6 & 40.4 & 13.5 \\
Raja & 204.8 & 19.5 & 372 & 17.8 & $1945 \dagger$ & $1037 \dagger$ & 14.6 & 24.5 & 42.2 & 11.3 \\
\hline average & 172.48 & 42.16 & 279.68 & 31.42 & & & 19.24 & 27.51 & 36.72 & 15.24 \\
normalized & 1 & 1 & 1.62 & 0.75 & & & 0.11 & 0.65 & 0.21 & 0.36
\end{tabular}

\section{Accordion Clocks}

A second problem we have tackled in TRaDe is the problem of the dynamic creation of threads. Many applications continuously create new threads: a browser updating a picture in a web page, an editor checking for modified files on disk, etc. To explain why this is a problem, we have to delve a little deeper in how data race are detected.

In parallel shared-memory programs there are two types of races: data races and synchronisation races. An example of a data race was already given in Figure 1

In Figure 2 we see the other type of race, a synchronisation race. A synchronisation race occurs when two threads perform a synchronisation operation (like obtaining a mutex, a $\mathrm{P}$ operation on a semaphore, ...) on the same synchronisation object in a non-determined order.

The set of operations that are performed by a thread between two consecutive synchronisation operations is called a segment (e.g. $S_{1}, S_{2}, S_{3}, S_{4}, \ldots$ in Figure [2). 


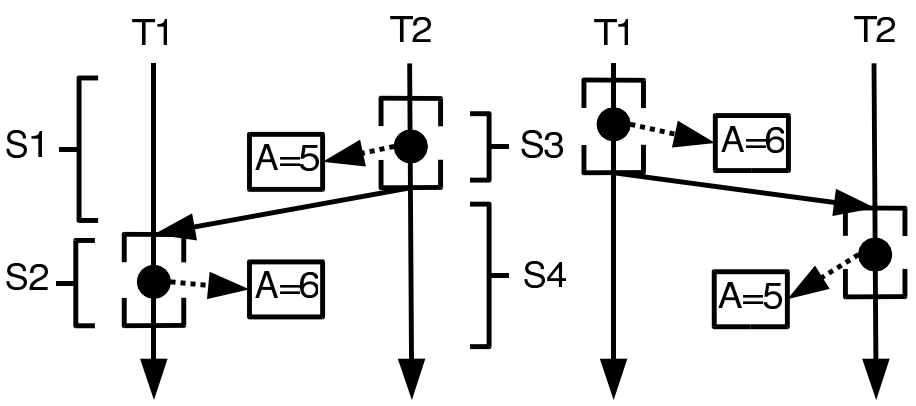

Fig. 2. The threads enter a mutex (indicated by the opening and closing brackets) before modifying the common variable. The final result of the execution is determined by which thread enters the mutex first. The order in which the threads access the mutex is indicated by the solid arrows.

Synchronisation primitives induce a partial order on the segments. For example, in Figure 2, thread $T_{2}$ is able to enter the mutex first. Thread $T_{1}$ must wait until $T_{2}$ leaves the mutex. Therefore, segment $S_{3}$ is ordered before $S_{2}$. We write $S_{3} \stackrel{\operatorname{syn}}{\rightarrow} S_{2}$.

In addition, segments are partially ordered simply by the fact that they are sequentially executed by their thread. Segment $S_{1}$ for example is executed before $S_{2}$ by thread $T_{1}$. We write $S_{1} \stackrel{s e q}{\rightarrow} S_{2}$.

Using these two partial orders, we can define the "execution order" on two segments $S_{x}$ and $S_{y}$ as the transitive closure of the union of the two previous relations:

$$
S_{x} \rightarrow S_{y} \equiv S_{x} \stackrel{\text { syn }}{\rightarrow} S_{y} \vee S_{x} \stackrel{\text { seq }}{\rightarrow} S_{y} \vee \exists S_{z} \cdot\left(S_{x} \rightarrow S_{z} \wedge S_{z} \rightarrow S_{y}\right)
$$

Two segments are said to be "parallel" if there is no execution order between them i.e.

$$
S_{x} \| S_{y} \equiv \neg\left(\left(S_{x} \rightarrow S_{y}\right) \vee\left(S_{y} \rightarrow S_{x}\right)\right)
$$

A data race occurs when operations from two parallel segments access a common variable and at least one operation is a write operation. If we define the set of locations written to resp. read from by a segment $S$ as $W(S)$ and $R(S)$ then a data race occurs between two segments, $S_{x}$ and $S_{y}$, when the following two conditions hold:

$$
\begin{gathered}
S_{x} \| S_{y} \\
\left(R\left(S_{x}\right) \cup W\left(S_{x}\right)\right) \cap W\left(S_{y}\right) \neq \emptyset \vee\left(R\left(S_{y}\right) \cup W\left(S_{y}\right)\right) \cap W\left(S_{x}\right) \neq \emptyset
\end{gathered}
$$

Checking in practice whether or not two segments are ordered can be done using a construct called a "vector clock" [12], [4], [5]. A vector clock is essentially just an array of integers (one for every thread in the program) that is updated each time a synchronisation operation occurs. A comparison function, 
$<$, is defined for vector clocks, $V_{1}$ and $V_{2}$, as follows

$$
V_{1}<V_{2} \equiv\left(\left(\forall i . V_{1}[i] \leq V_{2}[i]\right) \wedge V_{1} \neq V_{2}\right)
$$

It has the nice property of being a mapping of the execution order i.e. if $V C$ is a function producing the vector clock of a segment then the following holds:

$$
S_{x} \rightarrow S_{y} \equiv V C\left(S_{x}\right)<V C\left(S_{y}\right)
$$

In [3] it is proven that the size of a vector clock is proportional to the maximum number of parallel threads in the multi-threaded program. In general, we can't just reduce the size of the vector clock when a thread dies.

Why this is the case can be seen in Figure 3. We see a simple FTP-server which creates a new slave thread for each file request. Depending on the size of the file to transfer, the bandwidth of the network connection to the client and many other factors, this slave thread can take a variable amount of time to finish. When the slave thread has finished transferring the file, it is destroyed.

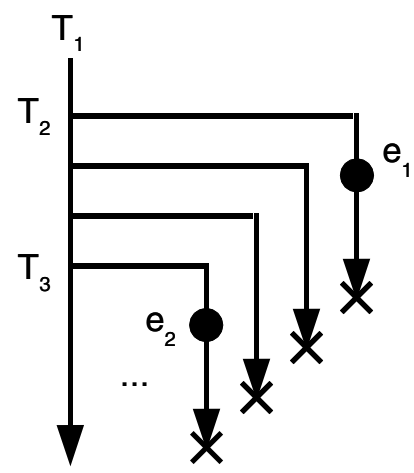

Fig. 3. An FTP-server spawning new threads for every file request.

Clearly, over a long period of time, only a small fraction of the slave threads will run concurrently. Still, the size of the vector clock will be equal to the total number of threads executed by the FTP-server. Indeed, if we would try to remove the information about the execution of thread $T_{2}$ when the thread has finished, we would run the risk of no longer detecting all data races.

Take for example event $e_{1}$ on thread $T_{2}$. Even when thread $T_{2}$ has finished execution, this event can still cause a race with another event $e_{2}$ on thread $T_{3}$. Indeed, if $T_{2}$ had run a little slower, $e_{2}$ would have been executed before $e_{1}$, which clearly constitutes a data race. The cause for this data race is that $T_{2}$ simply dies when it has finished its task without synchronizing with any other thread. Therefore, the risk for data races with event $e_{1}$ will continue to exist indefinitely and we can therefore never remove $T_{2}$ 's index from the vector clock. 
We have devised a new approach called 'accordion clocks' which allows us to surmount the problem of the ever growing size of vector clocks. The idea behind accordion clocks is simple. Suppose a thread has finished its execution. In general we cannot yet remove its index from the vector clocks since we might still need it to verify if another thread accesses the data structures touched by the dead thread in a not properly synchronized manner. If however we detect that a thread has ended and there are no data structures left which were touched by this thread then we can safely remove the index from the vector clock since no races are possible anymore.

Accordion clocks do just that. Initially, at program startup, all accordion clocks contain one index since only one thread is active. As threads are created during program execution the accordion clocks are expanded transparently to include new indices for the new threads. If a thread dies, this is noted and periodically all objects on the Java heap are checked to see whether there are any left which were once accessed by this deceased thread. If there are none left, the index corresponding to the deceased thread is removed transparently from all the accordion clocks in the system. The time overhead of this check is comparable to the time overhead of a full garbage collection cycle.

We have implemented accordion clocks in TRaDe and tested them using the benchmarks described in Table 3 The results can be seen in Figure 4. The total memory used for storing all data structures to do data race detection is shown as it evolves in time. We have divided the memory consumption in two part: the top part indicates the memory used for accordion clocks and vector clocks. The bottom part is used for other per object data structures to perform data race detection. On the left, we see the execution of the benchmarks without using accordion clocks. On the right accordion clocks are used.

It is clear that after a short period of time the memory used for storing accordion clocks becomes overwhelming. This prohibits searching data races in long running applications. When using accordion clocks, the memory consumed for storing clocks levels off after a while. For some applications, like Forte and Java2D, memory consumption seems to level off to a constant cost. For Swing, accordion clocks are clearly beneficial but some work will have to be done to stop the other data structures expanding.

\section{Conclusions}

In this article we have shown that, using a topological approach, it is possible to more efficiently detect data races in Java. We have implemented this technique in a Java virtual machine and have found it superior in execution speed to competing systems. Furthermore, we have found that a large part of the memory consumption while doing data race detection is due to storing vector clocks. We have solved the problem of the increasing size of vector clocks by introducing accordion clocks. Accordion clocks can grow and shrink as threads are created and destroyed while maintaining correct data race detection. 

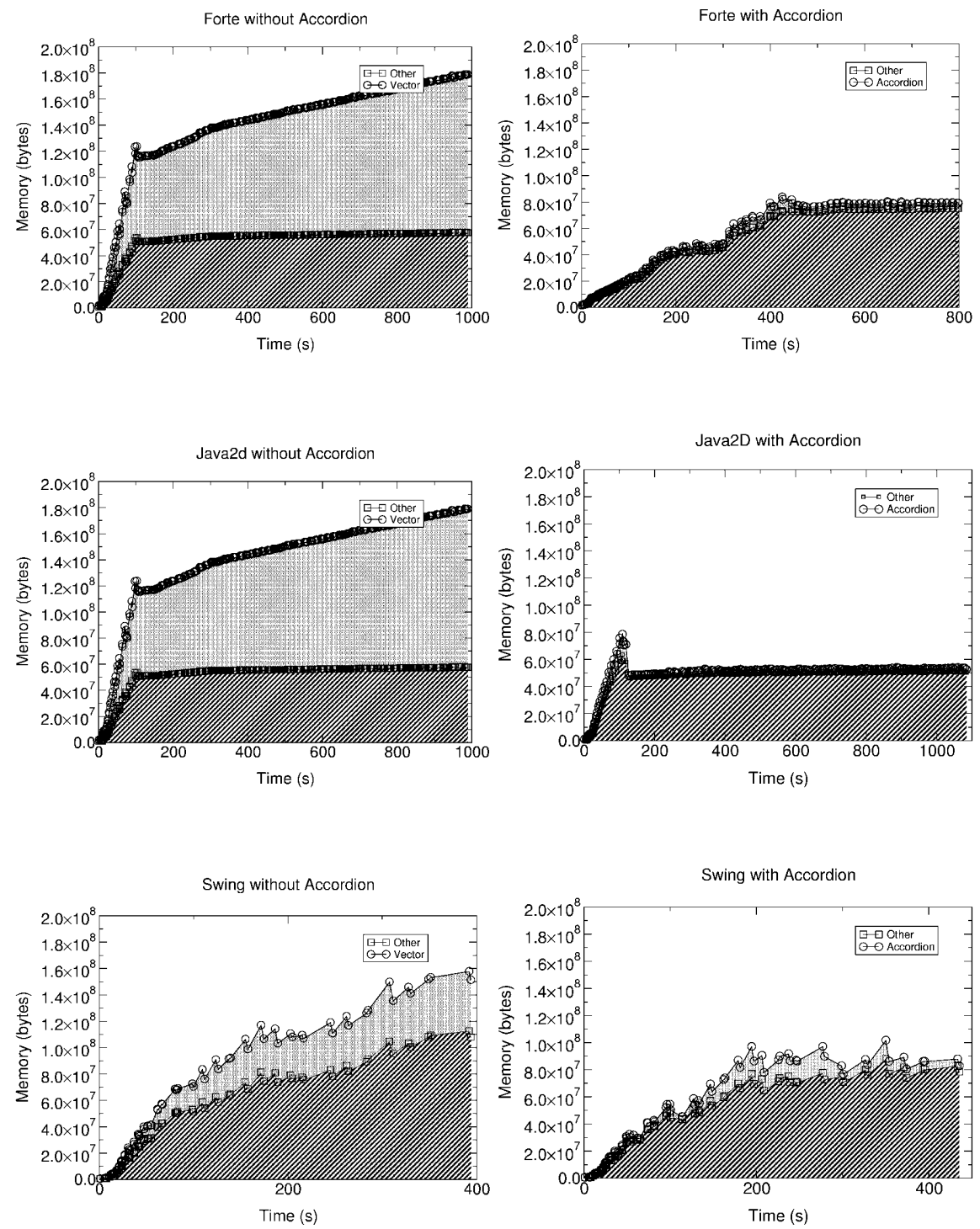

Fig. 4. Evolution of memory consumption during an execution of TRaDe without (on the left) accordion clocks and with (on the right) accordion clocks. 
Table 3.

\begin{tabular}{l|l} 
Name & Description \\
\hline SwingSet Demo & $\begin{array}{l}\text { A highly multi-threaded demo, included with the JDK } \\
1.2, \text { of the Swing widget set. It demonstrates buttons, } \\
\text { sliders, text areas, ... Every tab in the demo was clicked } \\
\text { twice during our tests. Immediately thereafter it was shut } \\
\text { down. } \\
\text { A highly multi-threaded demo, included with the JDK } \\
\text { Java2D Demo It demonstrates the features of the Java 2D drawing } \\
\text { libraries and is highly multi threaded. Every tab in the } \\
\text { demo was clicked 5 times. Immediately thereafter it was } \\
\text { shut down. } \\
\text { A full-blown Java IDE with object browsers, visual con- } \\
\text { struction of GUI code, ... To exercise the benchmark, we } \\
\text { created a small dialog while doing race detection. }\end{array}$
\end{tabular}

\section{Future Work}

We are currently exploring whether it is possible to do data race detection by instrumenting the bytecode of class files. Recently, a lot of research has been devoted to escape analysis [21]. This is a technique allowing to detect at compile time that an object will be local to a thread. We believe that this technique could be used to reduce the number of memory accesses that need to be observed thereby speeding up data race detection even further.

\section{Acknowledgments}

Mark Christiaens is supported by the GOA project 12050895. Our thanks go to Michiel Ronsse, with whom we had many stimulating discussions.

\section{References}

1. J. Aldrich, C. Chambers, E. G. Sirer, and S. Eggers. Static analyses for eliminating unnecessary synchronization from Java programs. In Static Analysis Symposium 99, September 1999.

2. Jeff Bogda and Urs Hölzle. Removing unnecessary synchronisation in Java . Technical report, Department of Computer Science, University of California, Santa Barbara, CA 93106, april 1999.

3. Bernadette Charron-Bost. Concerning the size of logical clocks in distributed systems. Information Processing Letters, 1(39):11-16, July 1991.

4. C.J. Fidge. Partial orders for parallel debugging. In Proceedings of the ACM SIGPLAN and SIGOPS Workshop on Parallel and distributed debugging, pages 183-194, May 1988.

5. Dieter Haban and Wolfgang Weigel. Global events and global breakpoints in distributed systems. In 21st Annual Hawaii International Conference on System Sciences, volume II, pages 166-175. IEEE Computer Society, January 1988. 
6. KL Group, 260 King Street East, Toronto, Ontario, Canada. Getting Started with JProbe.

7. Kuck \& Associates, Inc., 1906 Fox Drive, Champaign, IL 61820-7345, USA. Assure User's Manual, 2.0 edition, march 1999.

8. Tim Lindholm and Frank Yellin. The Java Virtual Machine Specification. The Java Series. Addison-Wesley, 1997.

9. R. H. B. Netzer. Race Condition Detection for Debugging Shared-Memory Parallel Programs. PhD thesis, University of Wisconsin-Madison, 1991.

10. Michiel Ronsse and Koen De Bosschere. JiTI: Tracing Memory References for Data Race Detection. In E. D'Hollander, F.J. Joubert, and U. Trottenberg, editors, Proceedings of ParCo97: Parallel Computing: Fundamentals, Applications and New Directions, volume 12 of Advances in Parallel Computing, pages 327-334, Bonn, February 1998. North Holland.

11. Stefan Savage, Michael Burrows, Greg Nelson, Patrick Sobalvarro, and Thomas Anderson. Eraser: A dynamic data race detector for multi-threaded programs. In Operating Systems Review, volume 31, pages 27-37. ACM, October 1997.

12. Reinhard Schwarz and Friedman Mattern. Detecting causal relationships in distributed computations: in search of the holy grail. Distributed Computing, 7(3):149174, 1994.

13. Bill Venners. Inside the Java Virtual Machine. McGraw-Hill, New York, New York, USA, second edition, 1999. 